\title{
The Impostor Phenomenon in Mental Health Professionals: Relationships Among Compassion Fatigue, Burnout, and Compassion Satisfaction
}

\author{
Pamela Clark $^{1}\left[\right.$ Chelsey Holden ${ }^{1} \cdot$ Marla Russell $^{1} \cdot$ Heather Downs ${ }^{1}$
}

Accepted: 20 April 2021 / Published online: 30 April 2021

(c) The Author(s), under exclusive licence to Springer Science+Business Media, LLC, part of Springer Nature 2021

\begin{abstract}
Imposter phenomenon is defined as a sense of intellectual fraudulence and an inability to internalize success and competency. Although imposter phenomenon has been noted in several populations, literature is sparse that focuses on mental health professionals. In addition, little is known about the relationships between imposter phenomenon, compassion fatigue, and compassion satisfaction for mental health workers. Using a survey design with a convenience sample of 158 mental health workers, this study found that imposter phenomenon was positively associated with compassion fatigue, as well as negatively associated with compassion satisfaction, when controlling for years of work and age. Further, the combination of lower levels of compassion satisfaction and higher levels of burnout predicted higher levels of imposter phenomenon. Implications and preventative measures are discussed.
\end{abstract}

Keywords Imposter phenomenon · Compassion fatigue $\cdot$ Compassion satisfaction $\cdot$ Burnout $\cdot$ Mental health professionals

Remaining mentally and emotionally sound in order to provide effective therapy services is a professional and ethical mandate for marriage and family therapists (MFTs; AAMFT Code of Ethics, 2015). Since there are any number of psychological and environmental factors that can impact the mental and emotional capacity of MFTs, it is essential for them to remain aware and informed of potential risks. One such risk could be imposter phenomenon (IP). Characterized by self-doubt, fears of being discovered as a fraud, and an inability to internalize successes (Clance \& Imes, 1978; Clance \& O'Toole, 1987; Clance, 1985a, 1985b), IP has been linked with several negative well-being outcomes including depression (Wang et al., 2018), anxiety (Hutchins \& Rainbolt, 2017), burnout (Villwock et al., 2016), and emotional instability (Rohrmann et al., 2016). In addition, research has established an association between IP and both gender stigma consciousness (Cokley et al., 2015) and higher levels of racial discrimination (Bernard et al., 2018;

Pamela Clark

pamela.rollins@usm.edu

1 School of Child and Family Sciences, The University of Southern Mississippi, 118 College Drive, \#5035, Hattiesburg, MS 39406, USA
Peteet et al., 2015) suggesting a link with larger gender and racial discourses and a possibility of rendering minority or marginalized clinicians at greater risk. However, despite these findings and the fact that high levels of IP with negative consequences have been noted in a variety of other health care professions (Gottlieb et al., 2020; Legassie et al., 2008; Oriel, 2004; Villwork et al., 2016), few studies have focused on whether IP is prevalent within the mental health field or what its impact might be on mental health clinicians (Royse-Roskowsky, 2010; Urwin, 2018).

In contrast, the debilitating impact of compassion fatigue on mental health professionals has been well documented (Adams et al., 2006; Can \& Watson, 2019; Martin-Cuellar, 2019; O'Brien \& Haaga, 2015; Wagaman et al., 2015). Defined as a form of caregiver burnout developed by those who work with traumatized individuals (Figley, 2002), the construct of compassion fatigue incorporates two components. The first component is burnout, defined as an occupational hazard more prevalent in situations where persons experience prolonged exposure to demanding interpersonal and emotional stressors (Adams et al, 2006; Maslach, 2001). Burnout can result in job absenteeism and high turnover, lower productivity effectiveness, and reduced job satisfaction (Maslach, 2001). A clinician may suffer from burnout without meeting criteria for compassion fatigue. Compassion 
fatigue surpasses simple burnout in that it also incorporates secondary traumatic stress (STS), a condition that mirrors post-traumatic stress and develops in response to exposure to knowledge about others' traumatizing events (Figley, 1995).

Outcomes for clinicians with compassion fatigue include negative emotional, cognitive, and behavioral changes (Craig \& Sprang, 2010; Figley, 1995) as well as depression and substance use disorders (Stamm, 2010). Since the risk of developing compassion fatigue appears to be higher for those possessing a strong empathic orientation with others' suffering, mental health clinicians may be particularly vulnerable (Adams et al., 2006). On the other hand, researchers have also noted that some mental health professionals flourish when working with traumatized individuals and experience higher levels of personal fulfillment and career satisfaction (Radey \& Figley, 2007). Labeled compassion satisfaction, this phenomenon has been defined as the sense of accomplishment and satisfaction resulting from doing one's job well and effectively (Stamm, 2010).

Although the negative impact of compassion fatigue on mental health clinicians is well-established, no research, to our knowledge, has explored the possible association between compassion fatigue and IP, another construct that evidence indicates impacts helping professionals adversely. As this is the case, it is unknown whether these two constructs are linked or how they might influence each other. Similarly, although an association has been noted between burnout and IP in other helping professions (Gottlieb et al., 2020; Villwock et al., 2016), this association has not been explored in the mental health field to our knowledge. Further, it is unknown whether any association exists between IP and compassion satisfaction. And finally, little is known about the simple prevalence of IP within the mental health profession. As these appear to be important avenues of study pursuant to promoting the well-being of mental health clinicians, including MFTs, the purpose of this study is to determine if mental health clinicians experience IP and, if so, how might it be associated with compassion fatigue, burnout and/or compassion satisfaction. The following literature review discusses in more detail the constructs of IP, compassion fatigue, burnout, and compassion satisfaction.

\section{Imposter Phenomenon}

Imposter phenomenon, also known as imposter syndrome or imposterism, was initially believed to be associated predominantly with high-functioning, high- achieving professional women who had experienced a great deal of success in their chosen fields but had difficulty internalizing their successes or attributing them to their own abilities (Clance \& Imes, 1978).. Although presenting as very confident and competent, underneath the façade they tended to discount any evidence of their own achievement and feared being unmasked as incompetent and unintelligent (Clance \& Imes). Mistakes were viewed as humiliating failures, and they focused on negative as opposed to positive feedback (Clance, 1985b).

Clance (1985b) also suggested there is an imposter profile comprised of six components. The first component is the impostor cycle in which sufferers engage in self-doubt, fear they cannot repeat their accomplishments, and dwell on past failures. They may experience doubt, worry, anxiety and fear around a project and either overwork and overprepare or procrastinate. Second is the need to be special or the best and, when they fall short of the best, exhibit a tendency to dismiss their very real talents. Third, the superwoman/superman aspect results in the need to do everything perfect and with ease. Fourth, a fear of failure associated with shame and humiliation that results in drastic measures to avoid making mistakes, including avoiding challenges or any situation in which there is a possibility of not excelling. Fifth, denial of competence and discounting praise resulting in an inability to accept positive feedback. Finally, fear of and guilt around success due to the fear of consequences stemming from family-of-origin or environmental messages.

Other researchers have suggested there may be IP typologies. For example, researchers Leonhardt and colleagues (2017), in their study of highly experienced executives, found evidence of two different types of imposters. The first group, labeled "true" imposters, exhibited the negative self-views generally associated with imposter phenomenon sufferers. However, the second group, labeled "strategic" imposters, exhibited less of the self-views characteristic of imposter phenomenon, leading the researchers to suggest that this group may have been engaging in deliberate selfpresentation designed to both lower expectations and appear more modest.

It has been speculated that vulnerability to IP may be embedded within social discourse on gender. Clance and Imes (1978) originally believed IP was a function of the internalization of societal sex role stereotypes purporting that women were not as competent as their male counterparts. The researchers suggested that the women's difficulty in attributing their success to their own ability made sense as, overall, women tended to have lower expectations of their ability to effectively complete tasks than men as well as a tendency to attribute their successes to unstable external factors and their failures to stable, internal factors (Deaux, 1976 as cited in Clance \& Imes, 1978). Although subsequent research has suggested IP is not gender specific, and men can also experience a sense of imposterism (Cowman \& Ferrari, 2002; Cromwell et al., 1990), prevalence findings are mixed. Some research has found females were more likely to score higher on IP measurements (Bernard et al, 2017; Cusak, et al., 2013; Legassie et al., 2008; Li et al., 2014; McGregor 
et al., 2008; Oriel et al., 2004) while other research found no gender differences (Caselman et al., 2005; Clark et al., 2014; Hutchins et al., 2018; Rohrmann et al., 2016; Sonnak $\&$ Towell, 2001). The relationship between gender and IP remains unclear and further research is needed.

However, a link has been established between the construct of gender stigma consciousness (GSC) and IP (Cokley et al., 2015). GSC is defined as chronic awareness of one's gender stigmatization status. Those who score high on GSC tend to believe that they will be judged based on their gender as opposed to their performance only (Pinel, 1999). Cokely et al. found that GSC positively predicted IP in both women and men, although it was stronger for women, and proposed that those scoring higher on GSC internalize feelings of being an intellectual fraud more often than those with lower scores. Although only one study, these findings suggest that how individuals view their gender may impact the likelihood of developing IP more than actual gender.

Other research suggests that IP, for people of color, is influenced by experiences of racial discrimination and minority stress. For example, McClain et al. (2016) found, in a sample of African American college students, a positive correlation between IP and levels of minority status stress, including racial discrimination, insensitive or marginalizing comments, and fears of not belonging. Similarly, Bernard et al. (2018) found a positive correlation between racial discrimination and IP and further suggested that experiences of racial discrimination can increase feelings of imposterism over time. In addition, Bernard et al. (2017), in a study of African American emerging adults, found an interaction between gender and racial discrimination and IP. Specifically, African American women who reported higher frequencies of racial discrimination incidents and lower levels of distress due to the discrimination were more likely to develop IP. Further, IP was found to serve as a moderator in the relationship between racial discrimination and mental health for African American students, in that impostorism increased the strength of the relationship between perceived racial discrimination and depression. (Cokley et al., 2015).

Research has also explored the prevalence and associated factors of imposter phenomenon in various populations. For example, a sample of assistant professors reported experiencing IP as emotionally unsettling and disruptive. Participants also described adverse psychological outcomes, such as stress and anxiety, and unfavorable work outcomes, such as avoiding opportunities, working harder, and procrastination (Hutchins \& Rainbolt, 2017). A larger study focusing on academic faculty found an association between avoidant coping styles and higher levels of IP scores, a pattern the researchers believed contributed to participants' low job satisfaction (Hutchins et al., 2018). For undergraduates, higher IP scores were associated with lower mental health scores and higher scores on test anxiety and perfectionism (Cusak et al., 2013; Wang et al., 2018) and higher scores on the Beck Depression Inventory (McGregor et al., 2008). Additionally, IP was linked with fear and worry as well as anxiety and guilt in response to praise or achievement in a sample of emerging adults (Lane, 2015). Villwock et al. (2016) found that imposter phenomenon was associated with women, burnout, exhaustion, emotional exhaustion, cynicism, and depersonalization for a sample of medical students.. For medical residents, higher rates of IP were positively associated with low self-esteem and institutional culture (Gottlieb et al., 2020) as well as burnout (Gottlieb et al., 2020; Legassie et al., 2008), depression, and anxiety (Oriel et al., 2004). Rohrmann et al. (2016) found imposter phenomenon to be associated with higher levels of anxiety, dysphoric moods, emotional instability, negative self-evaluation and perfectionism in a sample of managers.

Several factors have been found to be predictive of imposter phenomenon. For example, an anxious attachment style and narcissistic expectation/self-promotion predicted higher levels of IP (Gibson-Beverly \& Schwarts, 2008). Additionally, lack of parental care and parental overprotection were linked with higher imposter scores; however, only maternal care was negatively correlated with IP scores for males while both maternal and paternal care were negatively associated with IP for females ( $\mathrm{Li}$ et al., 2014). Similarly, parental overprotection or a lack of care in the paternal parenting style (Want \& Kleitman, 2006) and a greater degree of perceived parental control were strong predictors of IP (Sonnack \& Towell, 2001). Finally, higher scores on workaholism and mistrust and lower scores on self-esteem predicted higher IP scores (Ross \& Krukowski, 2003).

Factors found to act as buffers against the development of IP included social support, validation of success, positive affirmation, and both personal and shared reflections among physicians-in-training (Gottlieb et al., 2020). Higher levels of self-compassion, defined as kindness and understanding towards self (Neff, 2003), were negatively associated with higher levels of IP for first year undergraduates (Patzak et al, 2017). For adolescents, friend support served as a buffer against IP for males. Females, however, also needed the support of parents, teachers and classmates (Caselman et al., 2005). Peteet et al. (2015), in a sample of talented African American and Hispanic undergraduates, found that two components of racial identity, high affirmation and belonging, and higher levels of environmental mastery were identified as protective factors against IP. Hutchins et al. (2018) suggested using active coping styles may be a protective factor, based on findings that those experiencing IP tended toward avoidant coping styles that possibly contributed to low job satisfaction. Some studies have found that increased levels of experience in the profession were associated with lower levels of IP (Clark, 2014; Urwin, 2018) whereas others have not (Royse-Roskowsky, 2010). 


\section{Compassion Fatigue and Burnout}

Figley (1995) developed the concept of compassion fatigue (CF) in recognition of the constellation of symptoms often experienced by those who provide helping services to victims of traumatic events. Often used interchangeably with the term secondary traumatic stress (STS), compassion fatigue is characterized by symptoms mirroring PTSD in that the clinician reexperiences the client's traumatic event, engages in avoidance/numbing of reminders, and experiences persistent arousal as a result of being exposed to knowledge of the client's trauma (Adams et al., 2006). Additional symptoms could also include chronic physical exhaustion, emotional exhaustion, depersonalization, feelings of inequity, headaches, and weight loss (Figley, 1995). Since compassion fatigue is a form of caregiver burnout, sufferers often experience the characteristic symptoms of burnout, such as emotional exhaustion, depersonalization, and reduced personal accomplishment (Adams et al, 2006; Maslach, 2001). However, compassion fatigue differs from burnout in that it entails symptoms of STS and is generated by close contact and emotional engagement with traumatized individuals (Figley, 2002).

Compassion fatigue has been studied extensively in the field of mental health and a variety of associated factors have been identified. For example, Sprang et al. (2007), in a large sample of rural mental health professionals, found that higher levels of compassion fatigue were associated with female gender, higher caseload percentage of PTSD clients, and being a psychiatrist. Nelson-Gardell and Harris's (2003) findings indicated that child welfare workers who had a personal experience of child abuse or neglect were more at risk to develop CF with the greatest risk occurring for those who experienced more than one type of childhood trauma. Similarly, previous crime victimization and higher levels of empathy accounted for $45 \%$ of the variance in the development of compassion fatigue in a sample of trauma workers (MacRitchie \& Leibowitz, 2010). Low levels of emotional separation, a component of empathy that represents differentiation from patients, and high levels of occupational stress were predictive of higher CF scores in a sample of social workers in a trauma center (Badger et al., 2008). In their study of social workers and psychologists working with trauma victims, Craig and Sprang (2010) found that significant predictors of CF included a higher caseload percentage of PTSD clients and not using evidenced-based practices. Other factors found to be associated with compassion fatigue include burnout and being a new professional (Devilly et al., 2009); lower use of problem-focused coping strategies and higher use of emotion-focused coping (Zeidner, et al., 2013); maladaptive coping strategies (Thompson et al., 2014), ergonomic issues in the workplace (Cetrano et al., 2017; Thompson et al., 2014); impact of work on life (Centrano et al., 2017); higher levels of clinician empathy (MacRitchie \& Leibowitze, 2010) and lower levels of self-other awareness and emotion regulation (Wagaman et al., 2015).

Factors that may serve as buffers to developing compassion fatigue include vitality, defined as a subjective experience of self as autonomous, competent and engaged, for therapists with a personal trauma history (Martin-Cuellar et al., 2019). In addition, higher levels of social support, emotional intelligence, emotion management, and adaptive coping strategies were negatively correlated with compassion fatigue among health care professionals (Zeidner et al., 2013). Can and Watson's (2019) study of counselors-intraining found that higher levels of resilience and wellness were associated with lower levels of compassion fatigue. Other factors associated with lower levels of compassion fatigue include the practice of mindfulness (ConstantineBrown et al., 2017; Thompson et al., 2014); specialized trauma training (Sprang et al., 2007); higher levels of selfdifferentiation, professional self-esteem, and perceived marital quality (Finzi-Dottan \& Kormosh, 2018); possessing a sense of coherence (Linley \& Joseph, 2007) and use of an evidence-based therapy model (Craig \& Sprang, 2010).

Burnout appears to be the result of exposure to chronic emotional and interpersonal job stressors (Maslach, 2001) and is considered a component of compassion fatigue, although it can be present independently. Consisting of three dimensions: emotional exhaustion, depersonalization, and reduced personal accomplishment (Adams et al., 2006), burnout can result in job absenteeism and high turnover, lower productivity effectiveness, and reduced job satisfaction (Maslach, 2001). Factors facilitating the development of burnout include work overload and time pressure, role conflict and role ambiguity, clients' problem severity, lack of social support, lack of feedback and lack of autonomy (Maslach, 2001). Mental health clinicians reported higher levels of burnout than primary care clinicians (Imai et al., 2004), particularly those working in agencies and community mental health sites (Melamed et al., 2001; Sprang et al., 2007). In addition, the requirement to display emotions and empathy on the job accounted for additional variance in burnout scores above other work stressors (Zapf et al., 2001), and younger employees appeared most vulnerable to burnout (Maslach, 2001). Males often scored higher on the cynicism scale, and unmarried individuals (particularly males) appeared to be more susceptible to burnout, with singles reporting higher rates than divorced. Lower levels of hardiness, an external locus of control, low self-esteem and avoidant coping styles have also been found to be associated with burnout (Semmer, 1996). Higher levels of burnout have been linked to higher levels of impact of work on life and 
lower levels of employee trust (Cetrano et al., 2017); lower levels of self-differentiation (Finzi-Dottan et al., 2018); higher maladaptive coping, lower mindfulness attitudes, and lower levels of compassion satisfaction (Thompson et al., 2014); practicing in a rural setting (Sprang et al., 2007); lower levels of work engagement (Audin et al., 2017); and having a cognitive-behavioral orientation (Linley \& Joseph, 2007).

\section{Compassion Satisfaction}

Radey and Figley (2007) described the concept of compassion satisfaction as a feeling of fulfillment that clinicians can experience when working with traumatized clients and suggested that mental health professionals do not have to succumb to burnout and compassion fatigue but, instead, can flourish through experiencing the joy of helping others. The authors presented a model for developing compassion satisfaction based on positive psychology principles and suggested three interrelated elements: (a) keeping a positive attitude towards clients, (b) increasing stress management resources, and (c)increasing self-care through finding inspiration and happiness. Coupled with sound judgement, these components should constitute a pathway to compassion satisfaction.

Research appears to support Radey and Figley's (2007) suggestions. For example, Krause (2005), in a sample of mental health professionals working with adolescent sex offenders, found a significant positive correlation between self-care and levels of compassion satisfaction. Linley and Joseph's (2007) findings indicated more experience as a clinician, personal therapy, and supervision were associated with higher scores on compassion satisfaction in a sample of clinical and counseling psychologists. Cetrano et al. (2017), in their study of mental health staff, found that lower levels of perceived need of training, perceived quality of meetings, and perceived risks and uncertainties for the future were associated with higher levels of compassion satisfaction. Similarly, higher levels of self-differentiation, professional self-esteem, and perceived marital quality were found to be associated with higher scores on compassion satisfaction (Finzi-Dottan \& Kormosh, 2018). Higher levels of affective response, a component of empathy involving the neurological process of affect sharing, was positively correlated with higher levels of compassion satisfaction (Wagaman et al., 2015). Craig and Sprang's (2007) findings indicated that clinicians who were trained in the use of an evidenced-based model in a sample of trauma treatment therapists had higher levels of compassion satisfaction. Other factors associated with greater levels of compassion satisfaction include practicing self-care
(Krause, 2005); having more experience as a clinician, engaging in personal therapy and supervision (Liney \& Joseph, 2007); and using an evidenced-based model in one's clinical practice (Craig \& Sprang, 2010).

In summary, there is a dearth of literature that explores the construct of IP within the mental health field, despite evidence of its prevalence and negative impact in other health care professions. In addition, since both compassion fatigue and burnout have been identified as risks to the well-being and effectiveness of mental health clinicians, exploring whether there is an association among these constructs and how such an association might influence the risks of developing any of them appears to be an important area of study. This is particularly salient since we were unable to locate any research that addressed this topic. Finally, since compassion satisfaction is a possible positive outcome and source of resiliency for mental health clinicians, discovering the association, if any, between IP and compassion satisfaction also seems essential in promoting the well-being of therapists. This study proposes to address these gaps. Based on existing literature, the authors developed the following five hypotheses.

1. When controlling for years of work as a mental health professional and age, IP will be negatively associated with compassion satisfaction.

2. When controlling for years of work as a mental health professional and age, imposter phenomenon will be positively associated with compassion fatigue.

3. When controlling for years of work as a mental health professional and age, imposter phenomenon will be positively associated with burnout.

4. Imposter phenomenon will be negatively associated with years of work as a mental health professional.

5. Age, years of work, burnout, STS, and compassion satisfaction will be predictive of level of imposter phenomenon among mental health professionals.

\section{Method}

\section{Participants}

Participants included 158 individuals who identified themselves as mental health professionals. The majority were female (132; 83.5\%), Caucasian (134; 85\%), married (105; $66.5 \%$ ), and identified as marriage and family therapists (98; 62\%) working full time $(120 ; 76 \%)$. Ages ranged from 22 to 77 with a mean age of $41.24(S D=13.74)$. Years of work as a mental health professional ranged from one to 45 with a mean length of 12.06 years $(S D=10.31)$. See Table 1 for more demographic information. 
Table 1 Demographic characteristics of participants $(\mathrm{N}=158)$

\begin{tabular}{|c|c|c|}
\hline Characteristic & $n$ & $\begin{array}{l}\text { Percent } \\
\text { of sam- } \\
\text { ple }\end{array}$ \\
\hline \multicolumn{3}{|l|}{ Sex } \\
\hline Female & 132 & 83.5 \\
\hline Male & 24 & 15.2 \\
\hline Gender fluid & 2 & 1.3 \\
\hline \multicolumn{3}{|l|}{ Race/Ethnicity } \\
\hline White/Caucasian & 134 & 84.7 \\
\hline Hispanic/Latino & 5 & 3.2 \\
\hline Black/African American & 17 & 10.8 \\
\hline Other/prefer not to say & 2 & 1.3 \\
\hline \multicolumn{3}{|l|}{ Age } \\
\hline $20-25$ & 7 & 4.4 \\
\hline $26-30$ & 35 & 22.2 \\
\hline $31-35$ & 33 & 20.9 \\
\hline $36-40$ & 17 & 10.8 \\
\hline $41-45$ & 14 & 8.9 \\
\hline $46-50$ & 12 & 7.6 \\
\hline $51-55$ & 12 & 7.6 \\
\hline $56-60$ & 8 & 5.0 \\
\hline $61-65$ & 7 & 4.4 \\
\hline $65-70$ & 9 & 5.7 \\
\hline $71-75$ & 3 & 1.9 \\
\hline $75-80$ & 1 & 0.6 \\
\hline \multicolumn{3}{|l|}{ Marital status } \\
\hline Single/never married & 31 & 19.6 \\
\hline Married/domestic partnership & 105 & 66.4 \\
\hline Divorced & 20 & 12.7 \\
\hline Separated & 2 & 1.3 \\
\hline \multicolumn{3}{|l|}{ Mental health discipline } \\
\hline Marriage and family therapy & 98 & 62.0 \\
\hline Counseling & 43 & 27.2 \\
\hline School counseling & 7 & 4.4 \\
\hline Social work & 5 & 3.2 \\
\hline Other & 5 & 3.2 \\
\hline \multicolumn{3}{|l|}{ Employment status } \\
\hline Full time & 120 & 75.8 \\
\hline Part time & 24 & 15.2 \\
\hline Self-employed & 5 & 3.2 \\
\hline Student & 3 & 1.9 \\
\hline Retired & 2 & 1.3 \\
\hline Unemployed & 2 & 1.3 \\
\hline Other & 2 & 1.3 \\
\hline \multicolumn{3}{|c|}{$\begin{array}{l}\text { Years of work as mental health profes- } \\
\text { sional }\end{array}$} \\
\hline $1-5$ & 58 & 36.7 \\
\hline $6-10$ & 36 & 22.8 \\
\hline $11-15$ & 19 & 12.0 \\
\hline $16-20$ & 16 & 10.1 \\
\hline $21-25$ & 11 & 7.0 \\
\hline
\end{tabular}

Table 1 (continued)

\begin{tabular}{lll}
\hline Characteristic & $n$ & $\begin{array}{l}\text { Percent } \\
\text { of sam- } \\
\text { ple }\end{array}$ \\
\hline $26-30$ & 8 & 5.1 \\
$31-35$ & 3 & 1.9 \\
$36-40$ & 3 & 1.9 \\
$41-45$ & 4 & 2.5 \\
\hline
\end{tabular}

\section{Procedure}

This research project was approved by the Institutional Review Board at the authors' institution. Mental health professionals were invited to participate in an online research study via snowball sampling methods-all authors reached out to their contacts employed as mental health professionals to ask for participation in the research, and asked their contacts to also reach out to their contacts to recruit participants. The authors reached out to various mental health disciplines, including several state licensure boards for Licensed Professional Counselors and Licensed Marriage and Family Therapists. The authors sent email invitations to participate in a brief online survey available via Qualtrics survey platform. The authors also contacted state organizations for marriage and family therapists and professional counselors across the United States and asked these organizations to share the research invitation via e-mail and on their social media pages. Finally, the authors attended two state-level marriage and family therapy annual conferences (i.e., Mississippi's Marriage and Family Therapy Conference and Louisiana's Marriage and Family Therapy Conference) to recruit participants. Participants who clicked the link within the email were taken to an informed consent page where they could indicate they were 18 years of age or older and consent to participate in the research to continue to the demographic questions and instruments, or exit the survey.

\section{Instruments}

Participants answered several demographic questions, including age, gender, race/ethnicity, marital status, mental health discipline, employment status, and years of work as a mental health professional.

Participants completed the Professional Quality of Life Scale (ProQOL; Stamm, 2010). The ProQOL is a 30 -item self-report questionnaire answered on a 5-point Likert scale $(1=$ Never to $5=$ Very Often $)$. Participants are asked to indicate the number on the scale that honestly reflects their experiences over the past 30 days. Two constructs are measured by the ProQOL: compassion satisfaction and compassion fatigue. The compassion satisfaction scale measures the positive feelings and pleasure associated 
with completing one's job. A sample item is, "I get satisfaction from being able to help people." Two concepts are included in the measurement of compassion fatigue: burnout and STS. The Burnout scale measures exhaustion, hopelessness, and frustration related to completing one's job. A sample item is, "I feel worn out because of my work as a helper." The STS scale measures the effects of experiencing work-related exposure to the trauma of others. A sample item is, "I feel as though I am experiencing the trauma of someone I have helped." Each of these scales contain 10 items, and higher scores indicate higher endorsement of the construct. Burnout, STS, and compassion satisfaction scores range from 10 to 50; burnout and STS scores are combined to measure compassion fatigue, with scores ranging from 20 to 100 (Stamm, 2010). Cronbach's reliability in this study was 0.89 for compassion satisfaction, 0.78 for burnout, and 0.82 for STS. Stamm (2010) reported Cronbach's alphas of 0.88 for compassion satisfaction, 0.75 for burnout, and 0.81 for STS, as well as good construct validity for all three scales.

Participants also completed the Imposterism Scale (Leary et al., 2000). The Imposterism Scale is a seven-item self-report questionnaire answered on a 5-point Likert scale $(1=$ Not at all characteristic of me to $5=$ Extremely characteristic of me). Participants indicated the number of the scale that is most characteristic of themselves, with higher scores indicating greater endorsement of feelings of imposterism. A sample item is, "In some situations I feel like an imposter." Leary et al. (2000) reported Cronbach's alpha for the Imposterism scale to be 0.87 ; for this study, Cronnbach's alpha was excellent $(a=0.92)$. This brief, unidimensional measure of imposterism focuses on the core feelings of being inadequate and inauthentic, which are central to the experience of imposterism. This instrument has been found to be highly correlated with multidimensional measures of imposterism (i.e., Clance, 1985a, 1985b; Harvey \& Katz, 1985; Kolligian \& Sternberg, 1991) with strong evidence of construct validity (Leary et al., 2000).

\section{Statistical Analyses}

The data were analyzed using SPSS Statistics Version 26.0. The first three hypotheses were investigated using Pearson's partial correlations. Hypothesis 4 was investigated using Pearson's correlation. Hypothesis 5 was investigated using a standard multiple regression analysis; age, years of work, burnout, STS, and CS were entered as independent variables to predict the dependent variable, IP. A priori power analysis indicated a sample size of 135 participants was needed to achieve 0.95 power to detect a medium effect size. An alpha level of 0.05 was used for all analyses.

\section{Results}

\section{Data Cleaning and Preparation}

A total of 186 responses were received via the Qualtrics survey link. Twenty-seven cases (14.5\%) were removed due to completing only the demographic information and neither of the survey instruments. One case $(0.5 \%)$ was removed due to self-report that the participant was not a mental health professional, which was a requirement for inclusion in the study. The data were screened for outliers using Mahalanobis' distance; one case $(0.5 \%)$ was excluded ( $a=0.005$; Jennings \& Young, 1988) and was noted to have marked ' 1 ' for all answers. Cook's distance revealed no cases with undue influence on the data set. After the cleaning process, 157 participants $(84.4 \%)$ were included in data analysis. Descriptive statistics showed that the imposter phenomenon and Years of Work as a mental health professional variables demonstrated problematic skewness (>1.2). After square root transformations, skewness for imposter phenomenon was 0.868 and was 0.502 for Years of Work. Assumptions of linearity and normality were met for the correlational analyses. Assumptions of independence of residuals, homoscedasticity, linearity, multicollinearity, and normality were met for the multiple regression analysis.

Hypothesis 1 When controlling for years of work as a mental health professional and age, imposter phenomenon will be negatively associated with compassion satisfaction.

Results from a bivariate Pearson's correlation indicate that imposter phenomenon and compassion satisfaction have a statically significant negative linear relationship, $r(157)=-0.433, p<0.001$ (see Table 2). Pearson's partial correlation showed that the strength of the linear relationship between imposter phenomenon and compassion satisfaction decreases slightly when controlling for age and years of work as a mental health professional, $r_{\text {partial }}(153)=-0.417, p<0.001$ (see Table 3). Results indicate a medium effect size for this relationship (Cohen, 1988). The coefficient of determination $\left(r^{2}=0.173\right)$ shows that, when controlling for age and years of work, imposter phenomenon and compassion satisfaction share $17 \%$ of their variance with one another.

Hypothesis 2 When controlling for years of work as a mental health professional and age, imposter phenomenon will be positively associated with compassion fatigue.

Results from a bivariate Pearson's correlation indicate that imposter phenomenon and compassion fatigue 
Table 2 Correlations table

\begin{tabular}{llllllll}
\hline & IP & CS & CF & Burnout & STS & Years of work & Age \\
\hline IP & 1 & $-.433^{* *}$ & $.473^{* *}$ & $.508^{* *}$ & $.336^{* *}$ & $-.312^{* * *}$ & $-.300^{* *}$ \\
CS & $-.433^{* *}$ & 1 & & & & .134 & \\
CF & $.458^{* *}$ & $-.506^{* *}$ & 1 & & & -.135 \\
Burnout & $.499^{* *}$ & $-.650^{* *}$ & $.884^{* *}$ & 1 & & $-.188^{*}$ & \\
STS & $.318^{* *}$ & $-.258^{* *}$ & $.893^{* *}$ & $.579 * *$ & 1 & -.054 \\
Years of work & $-.281^{* *}$ & .106 & -.136 & $-.173^{*}$ & -.070 & 1 \\
Age & $-.291^{* *}$ & .140 & $-.216^{* *}$ & $-.232^{* *}$ & -.153 & $.786^{* *}$ & 1 \\
\hline
\end{tabular}

$N=157$

IP impostor phenomenon, CS compassion satisfaction, STS secondary traumatic stress

*Correlation is significant at the 0.05 level (2-tailed); **Correlation is significant at the 0.01 level (2-tailed). Correlations above the diagonal represent transformed values, and correlations below the diagonal represent non-transformed values

Table 3 Partial correlations table when controlling for age and years of work

\begin{tabular}{llllll}
\hline & IP & CS & CF & BO & STS \\
\hline IP & 1 & $-.417^{* *}$ & $.444^{* *}$ & $.467 * *$ & $.298^{* *}$ \\
CS & $-.413^{* *}$ & 1 & & & \\
CF & $.430^{* *}$ & $-.493^{* *}$ & 1 & & \\
BO & $.475^{* *}$ & $-.641^{* *}$ & $.879^{* *}$ & 1 & \\
STS & $.326^{* *}$ & $-.247^{* *}$ & $.891^{* *}$ & $.570^{* *}$ & 1 \\
\hline
\end{tabular}

$N=157$

$I P$ impostor phenomenon, $C S$ compassion satisfaction, $B O$ burnout, STS secondary traumatic stress

**Correlation is significant at the 0.01 level (2-tailed). Correlations above the diagonal represent transformed values, and correlations below the diagonal represent non-transformed values

have a statically significant positive linear relationship, $r(157)=0.473, p<0.001$ (see Table 2). Pearson's partial correlation showed that the strength of the linear relationship between imposter phenomenon and compassion fatigue decreases slightly when controlling for age and years of work as a mental health professional, $r_{\text {partial }}(153)=0.444 p<0.001$ (see Table 3 ). Results indicate a medium effect size for this relationship (Cohen, 1988). The coefficient of determination $\left(r^{2}=0.197\right)$ shows that, when controlling for age and years of work, imposter phenomenon and Compassion Fatigue share nearly 20\% of their variance with one another.

Hypothesis 3 When controlling for years of work as a mental health professional and age, imposter phenomenon will be positively associated with burnout.

Results from a bivariate Pearson's correlation indicate that imposter phenomenon and burnout have a statically significant positive linear relationship, $r(157)=0.508, p<0.001$ (see Table 2). Pearson's partial correlation showed that the strength of the linear relationship between imposter phenomenon and burnout decreases slightly when controlling for age and years of work as a mental health professional, $r_{\text {partial }}(153)=0.475 p<0.001$ (see Table 3 ). Results indicate a medium effect size for this relationship (Cohen, 1988). The coefficient of determination $\left(r^{2}=0.225\right)$ shows that, when controlling for age and years of work, imposter phenomenon and burnout share nearly $23 \%$ of their variance with one another.

Hypothesis 4 Imposter phenomenon will be negatively associated with years of work as a mental health professional.

Results from Pearson's correlation indicate that imposter phenomenon and years of work have a statically significant negative linear relationship, $r=-0.312, p<0.001$ (see Table 2). Results indicate a medium effect size for this relationship (Cohen, 1988). The coefficient of determination $\left(r^{2}=0.097\right)$ shows that imposter phenomenon and years of work share nearly $10 \%$ of their variance with one another.

Hypothesis 5 Age, years of work, burnout, compassion fatigue, and compassion satisfaction will be predictive of level of imposter phenomenon among mental health professionals.

Age, years of work, burnout, STS, and compassion satisfaction were entered as independent variables in a standard multiple regression to predict the dependent variable, imposter phenomenon. $R^{2}$ for the overall model was 0.333 with an adjusted $R^{2}$ of 0.311 , and $f^{2}$ of 0.499 , a large effect size according to Cohen (1988). The overall model was found to be significant, $F(5,151)=15.080, p<0.001$. In terms of unique contribution, compassion satisfaction contributed -0.199 to the model and was found to be statistically significant $(p=0.027)$, indicating that as compassion satisfaction increases, imposter phenomenon is predicted to 
Table 4 Summary of multiple regression analysis predicting IP

\begin{tabular}{lllll}
\hline Variable & $B$ & $\mathrm{SE}_{\mathrm{B}}$ & $\beta$ & $p$ \\
\hline Intercept & 3.832 & .710 & & .000 \\
Years of work & -.096 & .052 & -.201 & .065 \\
Age & -.002 & .005 & -.036 & .741 \\
CS & -.027 & .012 & -.199 & $.027^{*}$ \\
Burnout & .037 & .015 & .266 & $.014^{*}$ \\
STS & .015 & .011 & .114 & .174 \\
\hline
\end{tabular}

$B$ unstandardized regression coefficient, $S E_{B}$ Standard error of the coefficient, $\beta$ standard coefficient, $p$ significance threshold (set at .05), IP Impostor Phenomenon, CS compassion satisfaction, STS secondary traumatic stress

*Statistically significant

decrease. Burnout contributed 0.266 to the model and was found to be statistically significant $(p=0.014)$, indicating that as burnout increases, imposter phenomenon is predicted to also increase. Age, years of work, and STS were not found to be statistically significant individual contributors to the model (see Table 4).

\section{Discussion}

Our first hypothesis, that IP would be associated with compassion satisfaction when controlling for years of work as a mental health professional and age, was supported. IP was found to be negatively correlated with compassion satisfaction in our sample. Since compassion satisfaction entails a sense of accomplishment and satisfaction resulting from a sense of doing one's job well and effectively (Stamm, 2010), and the essence of IP is an inability to internalize accomplishments or believe that one does a good job (Clance \& Imes, 1978), this finding makes sense. IP may serve as a barrier to experiencing compassion satisfaction, and clinicians who experience feelings of imposterism may be inhibited from experiencing the full range of pleasure and enjoyment from working in this rewarding field. Alternately, it is possible that compassion satisfaction serves as a protective factor against imposter phenomenon for clinicians, and enjoyment of the job can help clinicians to feel a greater sense of belonging and validation within their profession, thus decreasing feelings of imposterism. The systemic forces in the therapist's external world are also worthy of consideration; clinicians who hold marginalized identities may have more difficulty feeling comfortable and safe in their work environment, which could in turn affect both CS and feelings of imposterism. It is worth noting that this correlational relationship, regardless of directionality, is not time-limited; results indicate this relationship holds true for clinicians in all stages of their career.
Similarly, our second and third hypotheses-that IP would be positively associated with compassion fatigue and burnout, regardless of age and years of work-were both supported. The significant relationship between IP and compassion fatigue as a whole, as well as burnout individually, is worth noting. Higher levels of IP suggest that an individual will be prone to have self-doubt and use negative work strategies, such as workaholism (Mir \& Kamal, 2018) and procrastination (Hutchins \& Rainbolt, 2017), which can contribute to burnout within the workplace. Higher levels of IP also indicate one would be less likely to practice selfcare strategies recommended to balance the demands of working with a traumatized population (Radley \& Figley, 2007), which can increase risk of experiencing STS. Also, those who experience compassion fatigue and burnout may be more likely to experience the feelings of not belonging and fear of being exposed as inadequate that are characteristic of IP. Therapists and supervisors alike should be aware of this association between IP and compassion fatigue and understand that the experience of one may contribute to experiencing the other as well; this relationship supersedes being experienced in the field and can affect new therapists as well as seasoned therapists. Additionally, therapists who hold marginalized identities may be at greater risk of experiencing the combination of compassion fatigue and IP symptomology due to the systemic and cultural forces influencing their stress levels, work environment, and personal lives (Bernard et al., 2018; Fernandez, 2020).

Our fourth hypothesis, that IP would be negatively associated with years of work for mental health professionals, was also supported. Again, consistent with research in other fields (Clark et al., 2014; Urwin, 2018), we found that IP decreases with years of work, suggesting that, as mental health workers become more familiar with the demands and requirements of their profession, experience successes, and perhaps obtain additional training, imposter feelings may be reduced and the identity of a mental health clinician assumed. This can be encouraging to therapists who are newer in the field and therapists of all identities, as it can be helpful to remember that one's feelings of newness or not belonging are common to many-if not all —who are beginning in a new setting or profession, regardless of background and experiences (Fernandez, 2020). More research is needed to understand the potentially complex relationship between years of work experience, clinicians' personal identities, and experiences of IP over time.

Our final hypothesis-that age, years of work, burnout, STS, and compassion satisfaction are predictive of level of imposter phenomenon for mental health professionals-was partially supported. Although the model was significant, only burnout and compassion satisfaction were found to be significant contributors to the model. To our knowledge, this is the first analysis that considered these factors as predictors 
of IP among mental health care workers. This information is helpful to identify mental health care professionals who may be at higher risk of experiencing IP and demonstrates the interrelated nature of burnout, compassion satisfaction, and IP. Specifically, our model shows the combination of lower levels of compassion satisfaction and higher levels of burnout are predictive of higher levels of IP, which provides support for self-care interventions addressing both constructs simultaneously. It would appear that work environments that foster appreciation of their clinicians and promote helpful self-care practices, thus decreasing risk of burnout and increasing feelings of compassion satisfaction, could effectively decrease risk of their clinicians experiencing IP.

Although not surprising, these results are significant as they represent the first study to explore the association between IP, compassion satisfaction, burnout, and secondary traumatic stress in a sample of mental health workers. The findings of this study have important implications for mental health professionals. IP appears to be one of the pathways through which clinicians could develop burnout, which can cause discomfort to clinicians and potentially result in attrition. Clinicians-particularly those newer to the field—and employers might consider assessing for levels of imposterism as a proactive, preventative measure for later problems, such as burnout and STS. Knowledge of this vulnerability may help clinicians to practice more self-care; attain additional training, particularly if they are working with a trauma population; focus on internalizing existing competencies and accomplishments; and practice self-compassion. Similarly, employers may want to focus on fostering an institutional culture of social support, validation of success, and positive affirmation shown to be protective factors against IP (Gottlieb et al., 2020). Additionally, Dancy and Brown (2011) called upon academic and professional organizations to provide more expansive and tailored professional development opportunities that include tools to address both the professional and personal challenges that marginalized groups experience with regard to IP as they navigate their career goals. Organizations can also promote authenticity for all members of their organizations and encourage individuals to use their personal stories as appropriate to embolden their professional voice, reach, and advocacy (Dancy \& Brown, 2011).

Clinical supervisors might consider including conversations about IP into self-of-the-therapist conversations to understand how the supervisee's identities, experiences, and beliefs shape his or her work experiences, and therefore may influence experiences of compassion satisfaction, burnout, and IP within the workplace. Having an avenue with one's supervisor to address one's feelings of inferiority or not belonging in the context of one's workplace identity may be a healthy, proactive approach to increase clinicians' awareness and decrease clinicians' risk of experiencing symptoms that could disrupt both their work and personal lives. Additionally, measures both within and outside of the workplace can be taken to fight against internal and external forces of IP. Intentionally surrounding oneself with a positive, supportive community, seeking out and maintaining a mentor relationship, finding work-life balance, and engaging in personalized and thoughtful self-care practices are key components to building and maintaining healthy views of oneself and building professional success (Fernandez, 2020). Also, taking the action of identifying and pursuing relevant avenues for personal advocacy, professional advocacy, community, and public education can serve as healthy outlets to discourage and dispel societal forces that may contribute to the therapist's experiences of marginalization or not belonging. For example, Fernandez (2020) shared about her specific efforts to organize relevant community groups, activities, and retreats for women of color in order to encourage one another's success and wellbeing in a professional context, as well as the fulfillment and belonging that came from her efforts.

Limitations for this study include the use of a convenience sample as opposed to a more randomized sampling. In addition, the majority of clinicians in this study were marriage and family therapists (62\%), female (83.5\%), and Caucasian $(85 \%)$. Additionally, this study did not account for several important descriptive features of clinicians, including gender identity, sexual orientation, licensure status, type(s) of license(s) held, and frequency of working with clients who have experienced trauma. Therefore, care should be taken in generalizing the results. Future studies should focus on expanding the base of mental health professions studied and compare the impact of IP on the different disciplines, expand the demographics of the population studied, and account for the influence that personal identities may have on one's experiences of CS, burnout, STS, and IP.

This study is significant for MFTs and other mental health clinicians in that it represents the first (to our knowledge) that explored the relationship between compassion satisfaction, compassion fatigue, and imposter phenomenon in a sample of mental health professionals. Higher scores on imposter phenomenon were found to be positively associated with higher scores of compassion fatigue, and lower scores of compassion satisfaction. In addition, imposter phenomenon was found to be associated with fewer years working in the field. More research is needed to understand the external factors that may influence these relationships; however, this is an important first step to understanding imposter phenomenon, compassion fatigue, and compassion satisfaction. 


\section{Declarations}

Conflict of interest The authors declare to the best of our knowledge there are no conflicts of interest or competing interests.

\section{References}

American Association of Marriage and Family Therapy (2015). Code of ethics. Code of Ethics (aamft.org).

Adams, R. E., Boscarino, J. A., \& Figley, C. R. (2006). Compassion fatigue and psychological distress among social workers: A validation study. American Journal of Orthopsychiatry, 76, 103-108. https://doi.org/10.1037/0002-9432.76.1.103

Audin, K., Burde, J., \& Ivtzan, I. (2017). Compassion fatigue, compassion satisfaction and work engagement in residential child care. Scottish Journal of Residential Child Care, 17, 1-25

Badger, K., Royse, D., \& Craig, C. (2008). Hospital social workers and indirect trauma exposure: An exploratory study of contributing factors. Health \& Social Work, 33, 63-71. https://doi.org/10. 1093/hsw/33.1.63

Bernard, D. L., Lige, Q. M., Willis, H. A., Sosoo, E. E., \& Neblett, E. W. (2017). The impostor phenomenon and mental health: The influence of racial discrimination and gender. Journal of Counseling Psychology, 64, 155-166. https://doi.org/10.1037/coun0 000197

Bernard, D. L., Hoggard, L. S., \& Neblett, E. W., Jr. (2018). Racial discrimination, racial identity, and impostor phenomenon: A profile approach. Cultural Diversity and Ethnic Minority Psychology, 24(1), 51-61. https://doi.org/10.1037/cdp0000161

Can, N., \& Watson, J. C. (2019). Individual and relational predictors of compassion fatigue among counselors-in-training. The Professional Counselor, 9, 284-297. https://doi.org/10.15241/nc/9.4.285

Caselman, T. D., Self, P. A., \& Self, A. (2005). Adolescent attributes contributing to the imposter phenomenon. Journal of Adolescence, 29, 395-495. https://doi.org/10.1016/j.adolescence.2005. 07.003

Cetrano, G., Tedeschi, F., Rabbi, L., Gosetti, G., Lora, A., Lamonaca, D., Manthorpe, J., \& Amaddeo, F. (2017). How are compassion fatigue, burnout, and compassion satisfaction affected by quality of working life? Findings from a survey of mental health staff in Italy. BMC Health Services Research, 17, 1-11. https://doi.org/ 10.1186/s12913-017-2726-x

Clance, P. R. (1985a). The impostor phenomenon. Peachtree.

Clance, P. R. (1985b). The Impostor Phenomenon: When success makes you feel like a fake. Peachtree Publishers.

Clance, P. R., \& Imes, S. A. (1978). The imposter phenomenon in high achieving women: Dynamics and therapeutic intervention. Psychotherapy: Theory, Research \& Practice, 15, 241-247. https:// doi.org/10.10937/h0086006

Clance, P. R., \& O'Toole, M. A. (1987). The imposter phenomenon: An internal barrier to empowerment and achievement. Women \& Therapy, 6, 51-64. https://doi.org/10.1300/J015V6N03_05

Clark, M., Vardeman, K., \& Barba, S. (2014). Perceived inadequacy: A study of the imposter phenomenon among college and research librarians. College and Research Libraries, 75, 255-271. https:// doi.org/10.5860/crl12-423

Cohen, J. (1988). Statistical power analysis for the behavioral sciences (2nd ed.). Lawrence Erlbaum Associates: Hillsdale, NJ.

Cokley, K., Awad, G., Smith, L., Jackson, S., Awosogba, O., Hurst, A., Stone, S., Blondeau, K., \& Roberts, D. (2015). The roles of gender stigma consciousness, impostor phenomenon and academic self-concept in the academic outcomes of women and men. Sex Roles, 73, 414-426. https://doi.org/10.1007/s11199-015-0516-7

Constantine Brown, J. L., Ong, J., Mathers, J. M., \& Decker, J. T. (2017). Compassion fatigue and mindfulness: Comparing mental health professionals and MSW student interns. Journal of Evidence-Informed Social Work, 14, 119-210. https://doi.org/10. 1080/23761407.2017.1302859

Cowman, S. E., \& Ferrari, J. R. (2002). “Am I for Real?” Predicting impostor feelings from self-handicapping and affective components. Social Behavior and Personality, 30, 119-126. https://doi. org/10.2224/sbp.2002.30.2.119

Craig, C. D., \& Sprang, C. (2010). Compassion satisfaction, compassion fatigue, and burnout in a national sample of trauma treatment therapists. Anxiety, Stress, \& Coping, 23, 319-339. https://doi.org/ 10.1080/10615800903085818

Cromwell, B., Brown, N. W., Sanchez-Huceles, J., \& Adair, F. L. (1990). The impostor phenomenon and personality characteristics of high school honor students. Journal of Social Behavior \& Personality, 5, 563-573

Cusak, C. E., Hughes, J. L., \& Nuhu, N. (2013). Connecting gender and mental health to imposter phenomenon feelings. Psi Chi Journal of Psychological Research, 8, 74-81. https://doi.org/10.24839/ 2164-8204.JN18.2.74

Dancy, T. E., II., \& Brown, M. C., II. (2011). The mentoring and induction of educators of color: Addressing the impostor syndrome in academe. Journal of School Leadership, 21, 607-634

Devilly, G. J., Wright, R., \& Varker, T. (2009). Vicarious trauma, secondary traumatic stress or simply burnout? Effect of trauma therapy on mental health professionals. Australian and New Zealand Journal of Psychiatry, 43, 373-385. https://doi.org/10.1080/ 00048670902721079

Fernandez, D. (2020). You're going to need a team: Community, mentoring, self-care, and other lessons from the McNair Scholars Program. In K. D. McKee \& D. A. Delgado (Eds.), Degrees of difference: Reflections of women of color on graduate school. University of Illinois Press: Urbina, IL.

Figley, C. R. (1995). Compassion fatigue as secondary traumatic stress disorder: An overview. In C. R. Figley (Ed.), Compassion fatigue: Coping with secondary traumatic stress disorder in those who treat the traumatized (pp. 1-20). Brunner-Routledge.

Figley, C. R. (2002). Compassion fatigue: Psychotherapists' chronic lack of self-care. Journal of Clinical Psychology. In Session, 58(11), 1433-1441. https://doi.org/10.1002/jclp.10090

Finzi-Dottan, R., \& Kormosh, M. B. (2018). The spillover of compassion fatigue into marital quality: A mediation model. Traumatology, 24, 113-122. https://doi.org/10.1037/trm0000137

Gibson-Beverly, G., \& Schwartz, J. P. (2008). Attachment, entitlement, and the impostor phenomenon in female graduate students. Journal in College Counseling, 11, 119-132. https://doi.org/10.1002/j. 2161-1882.2008.tb00029.x

Gottlieb, M., Chung, A., Battaglioli, N., Sebok-Syer, S. S., \& Kalatari, A. (2020). Impostor syndrome among physicians and physicians in training: A scoping review. Medical Education, 54, 116-124. https://doi.org/10.1111/medu.13956

Harvey, J. C., \& Katz, C. (1985). If I'm so successful, why do I feel like a fake? The impostor phenomenon. St. Martin's.

Hutchins, H. M., \& Rainbolt, H. (2017). What triggers imposter phenomenon among academic faculty? A critical incident study exploring antecedents, coping, and development opportunities. Human Resource Development International, 20, 194-214. https://doi.org/10.1080/13678868.2016.1248205

Hutchins, H. M., Penny, L. M., \& Sublett, L. W. (2018). What imposters risk at work: Exploring imposter phenomenon, stress coping, and job outcomes. Human Resource Development Quarterly, 29, 31-48. https://doi.org/10.1002/hrdg.21304 
Imai, H., Nakao, H., Tsuchiya, M., Kuroda, Y., \& Katoh, T. (2004). Burnout and work environments of public health nurses involved in mental health care. Occupational and Environmental Medicine, 61, 764-768. https://doi.org/10.1136/oem.2003.009134

Jennings, L. W., \& Young, D. M. (1988). Forecasting methods for institutional research. In B. Yancey (Ed.), New directions for institutional research: Applying statistics in institutional research (pp. 77-96). Jossey-Bass Publishers: San Fransico, CA.

Kolligian, J., Jr., \& Sternberg, R. J. (1991). Perceived fraudulence in young adults: Is there an "impostor syndrome"? Journal of Personality Assessment, 56, 308-326. https://doi.org/10.1207/s1532 7752jpa5602_10

Kraus, V. I. (2005). Relationship between self-care and compassion satisfaction, compassion fatigue, and burnout among mental health professionals working with adolescent sex offenders. Counseling and Clinical Psychology Journal, 2, 81-88

Lane, J. A. (2015). The imposter phenomenon among emerging adults transitioning into professional life: Developing a grounded theory. Adultspan Journal, 14, 114-128. https://doi. org/10.1002/adsp.12009

Leary, M. R., Patton, K. M., Orlando, A. E., \& Funk, W. W. (2000). The impostor phenomenon: Self-perceptions, reflected appraisals, and interpersonal strategies. Journal of Personality, 68(4), 725-756. https://doi.org/10.1111/1467-6494.00114

Legassie, J., Zibrowski, E. M., \& Goldszmidt, M. A. (2008). Measuring resident well-being: Impostorism and burnout syndrome in residency. Journal of General Internal Medicine, 23(7), 10901094. https://doi.org/10.1007/s11606-008-0536-X

Leonhardt, M., Bechtoldt, M. N., \& Rohrmann, S. (2017). All impostors aren't alike-Differentiating the impostor phenomenon. Frontiers in Psychology, 8, 1-10. https://doi.org/10.3389/fpsyg. 2017.01505

Li, S., Hughes, J. L., \& Thu, S. M. (2014). The links between parenting styles and imposter phenomenon. Psi Chi Journal of Psychological Research, 19, 50-57

Linley, P. A., \& Joseph, S. (2007). Therapy work and therapists' positive and negative well-being. Journal of Social and Clinical Psychology, 26, 385-403. https://doi.org/10.1521/jscp.2007. 26.3.385

MacRitchie, V., \& Leibowitz, V. (2010). Secondary traumatic stress, level of exposure, empathy and social support in trauma workers. South African Journal of Psychology, 40, 149-158. https:// doi.org/10.1177/008124631004000204

Martin-Cuellar, A., Lardier, D. T., Jr., Atencio, D. J., Kelly, R. J., \& Monanez, M. (2019). Vitality as a moderator of clinician history of trauma and compassion fatigue. Contemporary Family Therapy, 41, 408-419. https://doi.org/10.1007/s10591-019-09508-7

Maslach, C. (2001). What have we learned about burnout and health? Psychology and Health, 16, 607-611. https://doi.org/10.1080/ 08870440108405530

McClain, S., Beasley, S. T., Jones, B., Awosogba, O., Jacskson, S., \& Cokely, K. (2016). An examination of the impact of racial discrimination, ethnic identity, imposter feelings, and minority status stress on the mental health of Black college students. Journal of Multicultural Counseling and Development, 44, 101-117. https://doi.org/10.1002/jmcd.12040

McGregor, L. N., Gee, D. E., \& Posey, K. E. (2008). I feel like a fraud and it depresses me: The relation between the imposter phenomenon and depression. Social Behavior and Personality, 36, 43-48. https://doi.org/10.2224/sbp.2008.36.1.43

Melamed, Y., Szor, H., \& Bernstein, E. (2001). The loneliness of the therapist in the public outpatient clinic. Journal of Contemporary Psychotherapy, 31, 103-112. https://doi.org/10.1023/A: 1010213606443
Mir, I., \& Kamal, A. (2018). Role of workaholism and self-concept in predicting imposter feelings among employees. Pakistan Journal of Psychological Research, 23, 413-487

Neff, K. D. (2003). Self-compassion: An alternative conceptualization of a healthy attitude toward oneself. Self Identity, 2, 85-101. https://doi.org/10.1080/15298860309031

Nelson-Gardell, D., \& Harris, D. (2003). Child abuse history, secondary traumatic stress and childcare workers. Child Welfare, 82, $5-26$

O'Brien, J. L., \& Haaga, D. A. (2015). Empathic accuracy and compassion fatigue among therapist trainees. Professional Psychology: Research and Practice, 46, 414-420. https://doi.org/10.1037/ pro0000037

Oriel, K., Plane, M. B., \& Mundt, M. (2004). Family medicine residents and the Impostor Phenomenon. Family Medicine, 36, 248-252

Patzak, A., Kollmayer, M. \& Schober, B. (2017) Buffering impostor feelings with kindness: The mediating role of self-compassion between gender-role orientation and the impostor phenomenon. Frontiers in Psychology, 8, 1-12. https://doi.org/10.3389/fpsyg. 2017.01289

Pinel, E. C. (1999). Stigma consciousness: The psychological legacy of social stereotypes. Journal of Personality and Social Psychology, 76, 114-128. https://doi.org/10.1037/0022-3514.76.1.114

Peteet, B. J., Montgomery, L., \& Weekes, J. C. (2015). Predictors of imposter phenomenon among talented ethnic minority undergraduate students. The Journal of Negro Education, 84, 2, 175-186. https://doi.org/10.7709/jnegroeducation.84.2.0175

Radey, M., \& Figley, C. R. (2007). The social psychology of compassion. Clinical Social Work, 35, 207-214. https://doi.org/10.1007/ s10615-007-0087-3

Rohrmann, S., Bechtoldt, M. N., \& Leonhardt, M. (2016). Validation of the impostor phenomenon among managers. Frontiers in Psychology, 7, 1-11. https://doi.org/10.3389/fpsyg.2016.00821

Ross, S. R., \& Krukowski, R. A. (2003). The imposter phenomenon and maladapative personality: Type and trait characteristics. Personality and Individual Difference, 34, 477-484. https://doi.org/ 10.1016/S0191-8869(02)00067-3

Royse-Roskowsky, J. C. (2010). Imposter phenomenon and counselor self-efficacy: The impact of imposter feelings [Unpublished doctoral dissertation]. Ball State.

Semmer, N. (1996). Individual differences, work stress, and health. In M. J. Schabracq, J.A.M. Winnubst, \& C. L. Cooper (Eds.), Handbook of Work and Health Psychology, (pp.51-86). Wiley.

Sonnak, C., \& Towell, T. (2001). The impostor phenomenon in British university students: Relationships between self-esteem, mental health, parental rearing style and socioeconomic status. Personality and Individual Differences, 31, 863-874

Sprang, G., Clark, J. J., \& Whitt-Woosley, A. (2007). Compassion fatigue, compassion satisfaction, and burnout: Factors impacting a professional's quality of life. Journal of Loss and Trauma, 12, 259-280. https://doi.org/10.1080/15325020701238093

Stamm, B. H. (2010). The Concise ProQOL Manual. (2nd ed.). ProQOL.org.

Thompson, I. A., Amatea, E. S., \& Thompson, E. S. (2014). Personal and contextual predictors of mental health counselors' compassion fatigue and burnout. Journal of Mental Health Counseling, $36,48-77$

Urwin, J. (2018). Imposter phenomena and experience levels in Social Work: An initial investigation. British Journal of Social Work, 48, 1432-1446. https://doi.org/10.1093/bjsw/bcx109

Villwock, J., Sobin, L. B., Koester, L., \& Harris, T. M. (2016). Impostor syndrome and burnout among American medical students: A pilot study. International Journal of Medical Education, 7, 364-369. https://doi.org/10.5116/ijme.5801.eac4 
Wagaman, M. A., Geiger, J. M., Shockley, C., \& Segal, E. A. (2015). The role of empathy in burnout, compassion satisfaction, and secondary traumatic stress among social workers. Social Work, 60, 201-209. https://doi.org/10.1093/sw/swv014

Wang, K. T., Sheveleva, M. S., \& Permyakova, T. M. (2018). Imposter syndrome among Russian students: The link between perfectionism and psychological distress. https://doi.org/10.1016/j.paid. 2019.02.005

Want, J., \& Keitman, S. (2006). Impostor phenomenon and self-handicapping: Links with parenting styles and self-confidence. Personality and Individual Differences, 40, 961-971. https://doi.org/10. 1016/j.paid.2005.10.005
Zapf, D., Seifert, C., Schmutte, B., \& Mertini, H. (2001). Emotion work and job stressors and their effects on burnout. Psychological Health, 16, 527-546. https://doi.org/10.1080/088704401084055 25

Zeidner, M., Hadar, D., Matthews, G., \& Roberts, R. D. (2013). Personal factors related to compassion fatigue in health professionals. Anxiety, Stress \& Coping, 26, 595-609. https://doi.org/10.1080/ 10615806.2013.777045

Publisher's Note Springer Nature remains neutral with regard to jurisdictional claims in published maps and institutional affiliations. 